\title{
Research Article \\ Characterization of Multiplicative Lie Triple Derivations on Rings
}

\author{
Xiaofei Qi \\ Department of Mathematics, Shanxi University, Taiyuan 030006, China \\ Correspondence should be addressed to Xiaofei Qi; xiaofeiqisxu@aliyun.com
}

Received 28 March 2014; Accepted 16 June 2014; Published 9 July 2014

Academic Editor: Geraldo Botelho

Copyright ( 2014 Xiaofei Qi. This is an open access article distributed under the Creative Commons Attribution License, which permits unrestricted use, distribution, and reproduction in any medium, provided the original work is properly cited.

Let $\mathscr{R}$ be a ring having unit 1 . Denote by $\mathscr{Z}(\mathscr{R})$ the center of $\mathscr{R}$. Assume that the characteristic of $\mathscr{R}$ is not 2 and there is an idempotent element $e \in \mathscr{R}$ such that $a \mathscr{R} e=\{0\} \Rightarrow a=0$ and $a \mathscr{R}(1-e)=\{0\} \Rightarrow a=0$. It is shown that, under some mild conditions, a map $L: \mathscr{R} \rightarrow \mathscr{R}$ is a multiplicative Lie triple derivation if and only if $L(x)=\delta(x)+h(x)$ for all $x \in \mathscr{R}$, where $\delta: \mathscr{R} \rightarrow \mathscr{R}$ is an additive derivation and $h: \mathscr{R} \rightarrow \mathscr{Z}(\mathscr{R})$ is a map satisfying $h([[a, b], c])=0$ for all $a, b, c \in \mathscr{R}$. As applications, all Lie (triple) derivations on prime rings and von Neumann algebras are characterized, which generalize some known results.

\section{Introduction}

Let $\mathscr{R}$ be an associative ring with the center $\mathscr{Z}(\mathscr{R})$. For any element $a, b \in \mathscr{R}$, we set $[a, b]=a b-b a$. Recall that a map $L: \mathscr{R} \rightarrow \mathscr{R}$ is a multiplicative derivation or nonlinear derivation if $L(a b)=L(a) b+a L(b)$, for all $a, b \in \mathscr{R}$, is a multiplicative Lie derivation, if $L([a, b])=[L(a), b]+$ $[a, L(b)]$, for all $a, b \in \mathscr{R}$, and is a multiplicative Lie triple derivation, if $L([[a, b], c])=[[L(a), b], c]+[[a, L(b)], c]+$ $[[a, b], L(c)]$, for all $a, b, c \in \mathscr{R}$. Particularly, if $L$ is additive (linear), then above maps are, respectively, additive (linear) derivations, additive (linear) Lie derivations, and additive (linear) Lie triple derivations. We often omit "linear" for "linear derivations."

The structure of additive (linear) derivations and additive (linear) Lie (triple) derivations on rings or algebras has been studied by many authors. Brešar in [1] proved that every additive Lie derivation on a prime ring $\mathscr{R}$ with characteristic not 2 can be decomposed as $\tau+\zeta$, where $\tau$ is an additive derivation from $\mathscr{R}$ into its central closure and $\zeta$ is an additive map of $\mathscr{R}$ into the extended centroid $\mathscr{C}$ sending commutators to zero. Mathieu and Villena [2] showed that every linear Lie derivation on a $C^{*}$-algebra is standard, that is, can be decomposed as the form $\tau+h$, where $\tau$ is a derivation and $h$ is a central valued linear map vanishing at each commutator. In [3] Qi and Hou proved that the same is true for additive
Lie derivations of nest algebras on Banach spaces. Miers [4] showed that every linear Lie triple derivation on $\mathscr{M}$, a von Neumann algebra with no central summands of type $I_{1}$, is of the form $\tau+h$, where $\tau$ is a derivation and $h$ is a central valued linear map vanishing at every Lie triple products $[[A, B], C]$. Recently, Wang and Lu [5] described the structure of linear Lie triple derivations on $\mathcal{J}$-subspace lattice algebras. For other results, see [6-10] and the references therein.

For the study of multiplicative derivations and multiplicative Lie (triple) derivations, Daif [11] initially proved that each multiplicative derivation on a 2-torsion free prime ring containing a nontrivial idempotent is additive. Yu and Zhang [12] showed that every multiplicative Lie derivation on triangular algebras is the sum of an additive derivation and a map into its center sending commutators to zero, and, later, Ji et al. [13] generalized this result to the case of multiplicative Lie triple derivations. Assume that $\mathcal{N}$ is a nontrivial nest on a Banach space $X$ over the complex field which contains a nontrivial complemented element, and $\operatorname{Alg} \mathcal{N}$ is the associated nest algebra. Li and Fang in [14] obtained the same result as the above for multiplicative Lie triple derivations on $\operatorname{Alg} \mathcal{N}$.

The purpose of the present paper is to consider the problem of characterizing nonlinear Lie triple derivations on general rings. 
Let $\mathscr{R}$ be a ring having unit 1 and an idempotent element $e$, and let $\mathscr{Z}(\mathscr{R})$ denote the center of $\mathscr{R}$. Assume that the characteristic of $\mathscr{R}$ is not 2 and satisfies that $a \mathscr{R} e=\{0\} \Rightarrow$ $a=0$ and $a \mathscr{R}(1-e)=\{0\} \Rightarrow a=0$. Let $L: \mathscr{R} \rightarrow \mathscr{R}$ be a multiplicative Lie triple derivation. We show that if $e \mathscr{R} e$ and $(1-e) \mathscr{R}(1-e)$ do not contain nonzero central ideals, then $L(a+b)=L(a)+L(b)+\lambda_{a, b}$, for all $a, b \in \mathscr{R}$, where $\lambda_{a, b} \in \mathscr{Z}(\mathscr{R})$ is a central element depending on $a$ and $b$ (Theorem 1); furthermore, if $\mathscr{R}$ also satisfies that, for $a \in \mathscr{R}$, $[e a e, e \mathscr{R} e] \subseteq \mathscr{Z}(e \mathscr{R} e) \Rightarrow e a e \in \mathscr{Z}(e \mathscr{R} e)$, and $[(1-e) a(1-$ $e),(1-e) \mathscr{R}(1-e)] \subseteq \mathscr{Z}((1-e) \mathscr{R}(1-e)) \Rightarrow(1-e) a(1-e) \epsilon$ $\mathscr{Z}((1-e) \mathscr{R}(1-e))$, then $L(a)=\delta(a)+h(a)$, for all $a \in \mathscr{R}$, where $\delta: \mathscr{R} \rightarrow \mathscr{R}$ is an additive derivation and $h: \mathscr{R} \rightarrow$ $\mathscr{Z}(\mathscr{R})$ is a map satisfying $h([[a, b], c])=0$, for all $a, b, c \in$ $\mathscr{R}$ (Theorem 2). As applications, some characterizations of multiplicative (additive) Lie (triple) derivations on prime rings and von Neumann algebras are obtained, respectively (Corollaries 3-7).

\section{Main Results and Corollaries}

The following are our main results in this paper.

Theorem 1. Let $\mathscr{R}$ be a ring having unit 1 and an idempotent element e. Assume that the characteristic of $\mathscr{R}$ is not 2 and $\mathscr{R}$ satisfies the following two conditions:

(i) $a \mathscr{R} e=\{0\} \Rightarrow a=0$ and $a \mathscr{R}(1-e)=\{0\} \Rightarrow a=0$;

(ii) e Re and $(1-e) \mathscr{R}(1-e)$ do not contain nonzero central ideals.

Assume that $L: \mathscr{R} \rightarrow \mathscr{R}$ is a multiplicative Lie triple derivation. Then $L(a+b)=L(a)+L(b)+\lambda_{a, b}$, for all $a, b \in \mathscr{R}$, where $\lambda_{a, b} \in \mathscr{Z}(\mathscr{R})$ is a central element depending on $a$ and $b$.

Moreover, if the ring $\mathscr{R}$ in Theorem 1 also satisfies that,

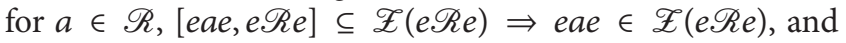
$[(1-e) a(1-e),(1-e) \mathscr{R}(1-e)] \subseteq \mathscr{Z}((1-e) \mathscr{R}(1-e)) \Rightarrow$ $(1-e) a(1-e) \in \mathscr{Z}((1-e) \mathscr{R}(1-e))$, then $L$ has more concrete form.

Theorem 2. Let $\mathscr{R}$ be a ring having unit 1 and an idempotent element e. Assume that the characteristic of $\mathscr{R}$ is not 2 and $\mathscr{R}$ satisfies the following two conditions:

(i) $a \mathscr{R} e=\{0\} \Rightarrow a=0$ and $a \mathscr{R}(1-e)=\{0\} \Rightarrow a=0$;

(ii) $e \mathscr{R e}$ and $(1-e) \mathscr{R}(1-e)$ do not contain nonzero central ideals;

(iii) for $a \in \mathscr{R}$, $[$ eae,$e \mathscr{R} e] \subseteq \mathscr{Z}(e \mathscr{R} e) \Rightarrow$ eae $\in \mathscr{Z}(e \mathscr{R} e)$, and $[(1-e) a(1-e),(1-e) \mathscr{R}(1-e)] \subseteq \mathscr{Z}((1-e) \mathscr{R}(1-$ $e)) \Rightarrow(1-e) a(1-e) \in \mathscr{Z}((1-e) \mathscr{R}(1-e))$.

Then a map $L: \mathscr{R} \rightarrow \mathscr{R}$ is a multiplicative Lie triple derivation if and only if $L(a)=\delta(a)+h(a)$, for all $a \in \mathscr{R}$, where $\delta: \mathscr{R} \rightarrow \mathscr{R}$ is an additive derivation and $h: \mathscr{R} \rightarrow \mathscr{Z}(\mathscr{R})$ is a map satisfying $h([[a, b], c])=0$, for all $a, b, c \in \mathscr{R}$.

Recall that a ring $\mathscr{R}$ is prime if, for any $a, b \in \mathscr{R}, a \mathscr{R} b=$ $\{0\}$ implies $a=0$ or $b=0$.
Apsult.

Applying Theorem 2 to prime rings, we have the following

Corollary 3. Let $\mathscr{R}$ be a prime ring having unit 1 and $a$ nontrivial idempotent, and let $L: \mathscr{R} \rightarrow \mathscr{R}$ be a map. If the characteristic of $\mathscr{R}$ is not 2 and e⿻Re, $(1-e) \mathscr{R}(1-e)$ are noncommutative, then the following two statements are equivalent.

(1) L is a multiplicative Lie triple derivation.

(2) There exist an additive derivation $\delta: \mathscr{R} \rightarrow \mathscr{R}$ and a map $h: \mathscr{R} \rightarrow \mathscr{Z}(\mathscr{R})$ satisfying $h([[a, b], c])=0$ for all $a, b, c \in \mathscr{R}$ such that $L(x)=\delta(x)+h(x)$ for all $x \in \mathscr{R}$.

Proof. Let $e \in \mathscr{R}$ be a nontrivial idempotent. It is obvious that $\mathscr{R}$ satisfies the condition (i) in Theorem 2 .

Claim. If $\mathcal{U}$ is a central ideal of a noncommutative prime ring $\mathscr{R}^{\prime}$, then $\mathscr{U}=\{0\}$.

Take any $u \in \mathcal{U}$. Since $\mathcal{U} \subseteq \mathscr{R}^{\prime}$ is central, we have $u a=$ au $\in \mathcal{U}$ for all $a \in \mathscr{R}^{\prime}$. Thus, for any $a, b \in \mathscr{R}^{\prime}$, one gets

$$
\begin{aligned}
u[a, b] & =u a b-u b a=u a b-a(u b) \\
& =u a b-(a u) b=u a b-(u a) b=0,
\end{aligned}
$$

and so

$$
u c[a, b]=u[c a, b]-u[c, b] a=0 \quad \forall a, b, c \in \mathscr{R}^{\prime} .
$$

Since $\mathscr{R}^{\prime}$ is noncommutative, there exist two elements $a_{0}, b_{0} \in$ $\mathscr{R}^{\prime}$ such that $\left[a_{0}, b_{0}\right] \neq 0$. It follows from the primeness of $\mathscr{R}^{\prime}$ that $u=0$. The claim holds.

Since $\mathscr{R}$ is prime, both $e \mathscr{R} e$ and $(1-e) \mathscr{R}(1-e)$ are prime. Thus, by the above claim, the condition (ii) in Theorem 2 is satisfied.

Now, for any fixed $a \in \mathscr{R}$, define two maps $\delta_{1}: e \mathscr{R} e \rightarrow$ $e \mathscr{R} e$ and $\delta_{2}:(1-e) \mathscr{R}(1-e) \rightarrow(1-e) \mathscr{R}(1-e)$, respectively, by

$$
\begin{gathered}
\delta_{1}(\text { exe })=[\text { eae }, \text { exe }], \\
\delta_{2}((1-e) x(1-e))=[(1-e) a(1-e),(1-e) x(1-e)],
\end{gathered}
$$

for all $x \in \mathscr{R}$. It is clear that both $\delta_{1}$ and $\delta_{2}$ are derivations. Posner in [9, Theorem 2] proved that, if $d$ is a derivation of a noncommutative prime $\mathscr{R}^{\prime}$ such that, for all $a \in \mathscr{R}^{\prime}, \operatorname{ad}(a)-$ $d(a) a$ is in the center of $\mathscr{R}^{\prime}$, then $d$ is the zero derivation. Thus, by [9, Theorem 2], for $a \in \mathscr{R}$, if $e \mathscr{R} e$ is noncommutative and $[e a e, e \mathscr{R} e] \subseteq \mathscr{Z}(e \mathscr{R} e)$, then $[e a e, e \mathscr{R} e]=0$; that is, eae $\epsilon$ $\mathscr{Z}(e \mathscr{R} e)$. Similarly, if $(1-e) \mathscr{R}(1-e)$ is noncommutative and $[(1-e) a(1-e),(1-e) \mathscr{R}(1-e)] \subseteq \mathscr{Z}((1-e) \mathscr{R}(1-e))$, then $(1-e) a(1-e) \in \mathscr{Z}((1-e) \mathscr{R}(1-e))$. Hence the condition (iii) in Theorem 2 is also satisfied.

Now, by Theorem 2, the corollary is true.

Let $\mathscr{B}(H)$ be the algebra of all bounded linear operators acting on a complex Hilbert space $H$. Recall that a von Neumann algebra $\mathscr{M}$ is a subalgebra of some $\mathscr{B}(H)$ satisfying 
$\mathscr{M}^{\prime \prime}=\left\{\mathscr{M}^{\prime}\right\}^{\prime}=\mathscr{M}$, where $\mathscr{M}^{\prime}=\{T: T \in \mathscr{B}(H)$ and $T A=$ $A T \forall A \in \mathscr{M}\}$ ([15]). For $A \in \mathscr{M}$, the central carrier of $A$, denoted by $\bar{A}$, is the intersection of all central projections $P$ such that $P A=0$. If $A$ is self-adjoint, then the core of $A$, denoted by $\underline{A}$, is $\sup \left\{S \in \mathscr{Z}(\mathscr{M}): S=S^{*}, S \leq A\right\}$. Particularly, if $A=P$ is a projection, it is clear that $\underline{P}$ is the largest central projection $\leq P$. A projection $P$ is core-free if $\underline{P}=0$. It is easy to see that $\underline{P}=0$ if and only if $\overline{I-P}=I([16])$.

Applying Theorem 2 to von Neumann algebras, we have the following corollary.

Corollary 4. Let $\mathscr{M}$ be a von Neumann algebra without central summands of type $I_{1}$ and $L: \mathscr{M} \rightarrow \mathscr{M}$ a map. Then the following statements are equivalent.

(1) L is a multiplicative Lie triple derivation.

(2) There exist an additive derivation $\delta: \mathscr{M} \rightarrow \mathscr{M}$ and a map $h: \mathscr{M} \rightarrow \mathscr{Z}(\mathscr{M})$ vanishing at each Lie triple product $[[A, B], C]$ such that $L(A)=\delta(A)+h(A)$, for all $A \in \mathscr{M}$.

Proof. Assume that $\mathscr{M}$ is a von Neumann algebra without central summands of type $I_{1}$. Then, by [16], there exists a nonzero core-free projection $P \in \mathscr{M}$ with $\bar{P}=I$. Fix such $P$ and note that $\bar{P}=\overline{I-P}=I$. It follows from the definition of the central carrier that both $\operatorname{span}\{T P(x): T \in \mathscr{M}, x \in H\}$ and $\operatorname{span}\{T(I-P)(x): T \in \mathscr{M}, x \in H\}$ are dense in $H$. So $A \mathscr{M} P=\{0\} \Rightarrow A=0$ and $A \mathscr{M}(I-P)=\{0\} \Rightarrow A=0$.

Brešar and Miers [17] proved that if $Z \in \mathscr{Z}(\mathscr{M})$ such that $Z \mathscr{M} \subseteq \mathscr{Z}(\mathscr{M})$, then $Z=0$. This implies that $\mathscr{M}$ has no nonzero central ideals. Note that $P \mathscr{M} P$ and $(I-P) \mathscr{M}(I-P)$ are also von Neumann algebras without central summands of type $I_{1}$. So both $P \mathscr{M} P$ and $(I-P) \mathscr{M}(I-P)$ have no nonzero central ideals.

Finally, for $A \in \mathscr{M}$, if $[P A P, P \mathscr{M} P] \subseteq \mathscr{Z}(P \mathscr{M} P)$ and $[(I-$ $P) A(I-P),(I-P) \mathscr{M}(I-P)] \subseteq \mathscr{Z}((I-P) \mathscr{M}(I-P))$, by the Kleinecke-Shirokov theorem $([18])$, both $[P A P, P T P]$ and $[(I-P) A(I-P),(I-P) T(I-P)]$ are central quasinilpotent for all $T \in \mathscr{M}$. Hence $[P A P, P T P]=[(I-P) A(I-P),(I-$ $P) T(I-P)]=0$ for all $T \in \mathscr{M}$; that is, $P A P \in \mathscr{Z}(P \mathscr{M} P)$ and $(I-P) A(I-P) \in \mathscr{Z}((I-P) \mathscr{M}(I-P))$.

Thus, if $\mathscr{M}$ has no central summands of type $I_{1}$, by what the above stated, $\mathscr{M}$ satisfies the corresponding assumptions (i)-(iii) in Theorem 2. By Theorem 2, the corollary is true.

Note that a multiplicative Lie derivation must be a multiplicative Lie triple derivation. So the following corollary is immediate.

Corollary 5. Let $M$ be a von Neumann algebra without central summands of type $I_{1}$ and $L: M \rightarrow M$ a map. Then the following statements are equivalent.

(1) L is a multiplicative Lie derivation.

(2) There exist an additive derivation $\delta: \mathscr{M} \rightarrow \mathscr{M}$ and $a$ map $h: \mathscr{M} \rightarrow \mathscr{Z}(\mathscr{M})$ vanishing at each commutator $[A, B]$ such that $L(A)=\delta(A)+h(A)$, for all $A \in \mathscr{M}$.
If $L$ in Corollaries 4 and 5 is additive, more can be said. In fact, a complete characterization of additive Lie (triple) derivations on any von Neumann algebras can be obtained, which is a slight generalization of the corresponding result in [4].

Corollary 6. Let $M$ be a von Neumann algebra and $L$ : $M \rightarrow \mathscr{M}$ an additive map. Then the following statements are equivalent.

(1) L is a Lie triple derivation.

(2) There exist an additive derivation $\delta: \mathscr{M} \rightarrow \mathscr{M}$ and an additive map $h: \mathscr{M} \rightarrow \mathscr{Z}(\mathscr{M})$ vanishing at each Lie triple product $[[A, B], C]$ such that $L(A)=\delta(A)+h(A)$, for all $A \in \mathscr{M}$.

Proof. Clearly, one only needs to check $(1) \Rightarrow(2)$. In the following assume that $L$ is an additive Lie triple derivation.

Take the central projection $Q \in \mathscr{M} \subseteq \mathscr{B}(H)$, so that, with respect to the space decomposition $H=Q H \oplus(I-Q) H$, $\mathscr{M}=\mathscr{M}_{1} \oplus \mathscr{M}_{2}$, where $\mathscr{M}_{1}$ is of type $I_{1}$ and $\mathscr{M}_{2}$ has no central summands of type $I_{1}$. Note that we may have $Q=0$. Then $L(A)$ can be decomposed as

$$
\begin{array}{r}
L(A)=Q L(A)+(I-Q) L(Q A)+(I-Q) L((I-Q) A) \\
\forall A \in \mathscr{M} .
\end{array}
$$

Claim. $L(\mathscr{Z}(\mathscr{M})) \subseteq \mathscr{Z}(\mathscr{M})$.

For any $Z \in \mathscr{Z}(\mathscr{M})$ and any $A, B \in \mathscr{M}$, we have $0=L(0)=L([[Z, A], B])=[[L(Z), A], B]$, and so $[L(Z), A] \in \mathscr{Z}(\mathscr{M})$ for all $A \in \mathscr{M}$. Hence $[L(Z), A]$ is central quasinilpotent, which implies $[L(Z), A]=0$ for all $A \in \mathscr{M}$. It follows that $L(Z) \in \mathscr{Z}(\mathscr{M})$. The claim holds.

Since $Q$ is a central projection and $Q \mathscr{M}$ is of type $I_{1}$, we have

$$
Q \mathscr{M} \subseteq \mathscr{Z}(\mathscr{M})
$$

By the above claim, $L(Q A) \in \mathscr{Z}(\mathscr{M})$, for all $A \in \mathscr{M}$, and so

$$
(I-Q) L(Q A)=L(Q A)-Q L(Q A) \in \mathscr{Z}(\mathscr{M}) \quad \forall A \in \mathscr{M} \text {. }
$$

Define a map $\Psi: \mathscr{M}_{2} \rightarrow \mathscr{M}_{2}$ by

$$
\begin{array}{r}
\Psi(B)=\left.L(0 \oplus B)\right|_{(I-Q) H}=\left.L((I-Q)(0 \oplus B))\right|_{(I-Q) H} \\
\forall B \in \mathscr{M}_{2} .
\end{array}
$$

Then, for any $A=A_{1} \oplus A_{2} \in \mathscr{M}=\mathscr{M}_{1} \oplus \mathscr{M}_{2}$, we have

$$
L(A)=Q L(A)+(I-Q) L(Q A)+\left(0 \oplus \Psi\left(A_{2}\right)\right) .
$$

It is easy to prove that $\Psi$ is an additive Lie triple derivation on $\mathscr{M}_{2}$. So, by Corollary 4 , there exist an additive derivation $\delta_{2}: \mathscr{M}_{2} \rightarrow \mathscr{M}_{2}$ and an additive map $h_{2}: \mathscr{M}_{2} \rightarrow \mathscr{Z}\left(\mathscr{M}_{2}\right)$ vanishing at each Lie triple product such that

$$
\Psi(B)=\delta_{2}(B)+h_{2}(B) \quad \forall B \in \mathscr{M}_{2} .
$$


Let $\delta=0 \oplus \delta_{2}$ and $h(A)=\mathrm{Q} L(A)+(I-Q) L(Q A)+$ $\left(0 \oplus h_{2}\left(\left.A\right|_{(I-Q) H}\right)\right)$. By (4)-(9), one gets that $\delta$ is an additive derivation on $\mathscr{M}$ and $h: \mathscr{M} \rightarrow \mathscr{Z}(\mathscr{M})$ is an additive map satisfying $h([[A, B], C])=0$ for all $A, B, C$ such that $L(A)=\delta(A)+h(A)$ holds for all $A \in \mathscr{M}$. Hence (2) is true and the proof is finished.

Particularly, for additive Lie derivations, we have the following corollary.

Corollary 7. Let $M$ be a von Neumann algebra and $L$ : $M \rightarrow M$ an additive map. Then the following statements are equivalent.

(1) L is a Lie derivation.

(2) There exist an additive derivation $\delta: \mathscr{M} \rightarrow \mathscr{M}$ and an additive map $h: \mathscr{M} \rightarrow \mathscr{Z}(\mathscr{M})$ vanishing at each commutator such that $L(A)=\delta(A)+h(A)$ for all $A \in$ $\mathscr{M}$.

\section{The Proof of Main Results}

In this section, we will give proofs of our main results, Theorems 1 and 2.

In the sequel, assume that $\mathscr{R}$ is a unital ring and containing an idempotent $e$ satisfying $a \mathscr{R} e=\{0\} \Rightarrow a=0$ and $a \mathscr{R}(1-e)=\{0\} \Rightarrow a=0$. It is clear that $e \neq 0,1$. Write $e_{1}=e$ and $e_{2}=1-e$. Then $\mathscr{R}$ can be written as $\mathscr{R}=\mathscr{R}_{11}+\mathscr{R}_{12}+\mathscr{R}_{21}+\mathscr{R}_{22}$, where $\mathscr{R}_{i j}=e_{i} \mathscr{R} e_{j}(i, j \in\{1,2\})$.

We first give several lemmas, which are needed to prove the main results.

Lemma 8 (see [3, Lemma 3.1]). The center of $\mathscr{R}$ is

$$
\begin{aligned}
\mathscr{Z}(\mathscr{R})=\left\{z_{11}+z_{22}\right. & : z_{11} \in \mathscr{R}_{11}, z_{22} \in \mathscr{R}_{22}, \\
z_{11} a_{12} & =a_{12} z_{22}, \\
z_{22} a_{21} & \left.=a_{21} z_{11} \forall a_{12} \in \mathscr{R}_{12}, a_{21} \in \mathscr{R}_{21}\right\} .
\end{aligned}
$$

By Lemma 8 , it is easily seen that if $z_{11}+z_{22} \in \mathscr{Z}(\mathscr{R})$, then $z_{11} \in \mathscr{Z}\left(\mathscr{R}_{11}\right)$ and $z_{22} \in \mathscr{E}\left(\mathscr{R}_{22}\right)$.

Lemma 9. Let $a \in \mathscr{R}$. If $a b_{12}=b_{12} a$ and $a b_{21}=b_{21} a$ hold for all $b_{12} \in \mathscr{R}_{12}$ and all $b_{21} \in \mathscr{R}_{21}$, then $a \in \mathscr{Z}(\mathscr{R})$.

Proof. Write $a=a_{11}+a_{12}+a_{21}+a_{22}$. Since $a b_{12}=b_{12} a$ for all $b_{12} \in \mathscr{R}_{12}$, we have

$$
a_{11} b_{12}+a_{21} b_{12}=b_{12} a_{21}+b_{12} a_{22} \text {. }
$$

Multiplying $e_{1}$ and $e_{2}$ from the left and right in the equation, one gets $a_{11} b_{12}=b_{12} a_{22}$. Multiplying $e_{2}$ from both sides in the equation, one has $a_{21} b_{12}=0$; that is, $a_{21} b e_{2}=0$ for all $b \in \mathscr{R}$. This implies $a_{21}=0$.

Similarly, from the relation $a b_{21}=b_{21} a$ for all $b_{21} \in \mathscr{R}_{21}$, one can prove $a_{22} b_{21}=b_{21} a_{11}$ and $a_{12}=0$. It follows from Lemma 8 that $a=a_{11}+a_{22} \in \mathscr{Z}(\mathscr{R})$.

Lemma 10. Let $a \in \mathscr{R}$. If $\left[\left[a, t_{i j}\right], e_{j}\right]=0$ holds for all $t_{i j} \in$ $\mathscr{R}_{i j}(1 \leq i \neq j \leq 2)$, then $e_{1} a e_{1}+e_{2} a e_{2} \in \mathscr{Z}(\mathscr{R})$.
Proof. For $1 \leq i \neq j \leq 2$, since $\left[\left[a, t_{i j}\right], e_{j}\right]=0$, we have $e_{i} a e_{i} t_{i j}=t_{i j} e_{j} a e_{j}$ for all $t_{i j} \in \mathscr{R}_{i j}$. It follows from Lemma 8 that $e_{1} a e_{1}+e_{2} a e_{2} \in \mathscr{Z}(\mathscr{R})$.

Lemma 11. For $a \in \mathscr{R}$, if $a \in \mathscr{Z}(\mathscr{R}) \cap \mathscr{R}_{i i}(i=1,2)$, then $a=0$.

Proof. For $i \in\{1,2\}$, assume that $a \in \mathscr{Z}(\mathscr{R}) \cap \mathscr{R}_{i i}$. So $a e_{i} b e_{j}=e_{i} b e_{j} a=0$ for all $b \in \mathscr{R}$, where $j \neq i$. It follows from the assumption on $\mathscr{R}$ that $a=a e_{i}=0$.

Lemma 12 (see [19, Lemma 4]). Let $\mathscr{R}^{\prime}$ be a ring having unit 1 and an idempotent element e. Assume that $\mathscr{R}^{\prime}$ satisfies that $a \mathscr{R}^{\prime} e=\{0\}$, which implies that $a=0$. For $a \in \mathscr{R}^{\prime}$, if exeae $=$ eaexe for all $x \in \mathscr{R}^{\prime}$, then there exists an element $\lambda \in \mathscr{Z}\left(\mathscr{R}^{\prime}\right)$ such that eae $=\lambda$ e.

Applying Lemma 12 to our ring $\mathscr{R}$, we get that, for any $a_{i i} \in \mathscr{R}_{i i}$, if $e_{i} x a_{i i}=a_{i i} x e_{i}$ holds for all $x \in \mathscr{R}$, then there exists $\lambda_{i} \in \mathscr{Z}(\mathscr{R})$ such that $a_{i i}=\lambda_{i} e_{i}, i=1,2$.

Now, we are in the position to give the proofs of Theorems 1 and 2.

Proof of Theorem 1. We will prove the theorem by a series of claims.

Claim 1. $L(0)=0$.

By the definition of $L$, we have

$$
\begin{aligned}
L(0) & =L([[0,0], 0])=[[L(0), 0], 0] \\
& +[[0, L(0)], 0]+[[0,0], L(0)]=0 .
\end{aligned}
$$

Claim 2. $e_{1} L\left(e_{i}\right) e_{1}+e_{2} L\left(e_{i}\right) e_{2} \in \mathscr{Z}(\mathscr{R}), i=1,2$.

Let $1 \leq i \neq j \leq 2$. For any $a_{i j} \in \mathscr{R}_{i j}$ and $e_{i}$, we have

$$
\begin{aligned}
L\left(a_{i j}\right)= & L\left(\left[\left[a_{i j}, e_{i}\right], e_{i}\right]\right) \\
= & {\left[\left[L\left(a_{i j}\right), e_{i}\right], e_{i}\right]+\left[\left[a_{i j}, L\left(e_{i}\right)\right], e_{i}\right] } \\
& +\left[\left[a_{i j}, e_{i}\right], L\left(e_{i}\right)\right] \\
= & L\left(a_{i j}\right) e_{i}-2 e_{i} L\left(a_{i j}\right) e_{i}+e_{i} L\left(a_{i j}\right)+a_{i j} L\left(e_{i}\right) e_{i} \\
& -a_{i j} L\left(e_{i}\right)+e_{i} L\left(e_{i}\right) a_{i j}-a_{i j} L\left(e_{i}\right)+L\left(e_{i}\right) a_{i j} .
\end{aligned}
$$

Multiplying by $e_{i}$ and $e_{j}$ from the left and the right in the above equation, respectively, one gets $2 e_{i} L\left(e_{i}\right) e_{i} a_{i j}=$ $2 a_{i j} e_{j} L\left(e_{i}\right) e_{j}$ for all $a_{i j} \in \mathscr{R}_{i j}$, which implies

$$
e_{i} L\left(e_{i}\right) e_{i} a_{i j}=a_{i j} e_{j} L\left(e_{i}\right) e_{j} \quad \forall a_{i j} \in \mathscr{R}_{i j},
$$

since the characteristic of $\mathscr{R}$ is not 2 .

Similarly, for any $a_{j i} \in \mathscr{R}_{j i}$, by the relation $L\left(a_{j i}\right)=$ $L\left(\left[\left[a_{j i}, e_{i}\right], e_{i}\right]\right)$, one can check that

$$
a_{j i} e_{i} L\left(e_{i}\right) e_{i}=e_{j} L\left(e_{i}\right) e_{j} a_{j i} \quad \forall a_{j i} \in \mathscr{R}_{j i} .
$$

Applying Lemma 8 to (14) and (15), one obtains $e_{i} L\left(e_{i}\right) e_{i}+$ $e_{j} L\left(e_{i}\right) e_{j} \in \mathscr{E}(\mathscr{R})$ for $1 \leq i \neq j \leq 2$. The claim holds. 
Now let $\delta(a)=L(a)-\left[a, e_{1} L\left(e_{1}\right) e_{2}-e_{2} L\left(e_{1}\right) e_{1}\right]$ for all $a \in \mathscr{R}$. Then $\delta: \mathscr{R} \rightarrow \mathscr{R}$ is also a multiplicative Lie triple derivation and satisfies $\delta\left(e_{1}\right) \in \mathscr{Z}(\mathscr{R})$ as Claim 2. Thus, without loss of generality, we may assume

$$
L\left(e_{1}\right) \in \mathscr{Z}(\mathscr{R}) .
$$

Claim 3. $L\left(e_{2}\right) \in \mathscr{Z}(\mathscr{R})$.

By Claim $2, e_{1} L\left(e_{2}\right) e_{1}+e_{2} L\left(e_{2}\right) e_{2} \in \mathscr{Z}(\mathscr{R})$. So we only need to check $e_{1} L\left(e_{2}\right) e_{2}=e_{2} L\left(e_{2}\right) e_{1}=0$. In fact, by (16), we have

$$
\begin{aligned}
0 & =L\left(\left[\left[e_{2}, e_{1}\right], e_{1}\right]\right) \\
& =\left[\left[L\left(e_{2}\right), e_{1}\right], e_{1}\right]+\left[\left[e_{2}, L\left(e_{1}\right)\right], e_{1}\right]+\left[\left[e_{2}, e_{1}\right], L\left(e_{1}\right)\right] \\
& =\left[\left[L\left(e_{2}\right), e_{1}\right], e_{1}\right]=e_{2} L\left(e_{2}\right) e_{1}+e_{1} L\left(e_{2}\right) e_{2},
\end{aligned}
$$

which implies $e_{1} L\left(e_{2}\right) e_{2}=e_{2} L\left(e_{2}\right) e_{1}=0$. Hence $L\left(e_{2}\right)=$ $e_{1} L\left(e_{2}\right) e_{1}+e_{2} L\left(e_{2}\right) e_{2} \in \mathscr{Z}(\mathscr{R})$.

Claim 4. $L\left(\mathscr{R}_{i j}\right) \subseteq \mathscr{R}_{i j}, 1 \leq i \neq j \leq 2$.

Here, we only give the proof for $L\left(\mathscr{R}_{12}\right) \subseteq \mathscr{R}_{12}$. The proof for the other inclusion $L\left(\mathscr{R}_{21}\right) \subseteq \mathscr{R}_{21}$ is similar.

For any $a_{12} \in \mathscr{R}_{12}$, by (16), (13) can be reduced to

$$
\begin{aligned}
L\left(a_{12}\right) & =L\left(a_{12}\right) e_{1}-2 e_{1} L\left(a_{12}\right) e_{1}+e_{1} L\left(a_{12}\right) \\
& =e_{1} L\left(a_{12}\right) e_{2}+e_{2} L\left(a_{12}\right) e_{1} .
\end{aligned}
$$

Taking any $b_{12} \in \mathscr{R}_{12}$ and any $c \in \mathscr{R}$, one obtains

$$
\begin{aligned}
0= & L\left(\left[\left[a_{12}, b_{12}\right], c\right]\right) \\
= & {\left[\left[L\left(a_{12}\right), b_{12}\right], c\right]+\left[\left[a_{12}, L\left(b_{12}\right)\right], c\right] } \\
& +\left[\left[a_{12}, b_{12}\right], L(c)\right] \\
= & {\left[\left[L\left(a_{12}\right), b_{12}\right]+\left[a_{12}, L\left(b_{12}\right)\right], c\right], }
\end{aligned}
$$

which implies $\left[L\left(a_{12}\right), b_{12}\right]+\left[a_{12}, L\left(b_{12}\right)\right] \in \mathscr{Z}(\mathscr{R})$. Note that

$$
\begin{aligned}
{\left[a_{12}, L\left(b_{12}\right)\right]=} & {\left[\left[e_{1}, a_{12}\right], L\left(b_{12}\right)\right] } \\
= & L\left(\left[\left[e_{1}, a_{12}\right], b_{12}\right]\right) \\
& -\left[\left[L\left(e_{1}\right), a_{12}\right], b_{12}\right] \\
& -\left[\left[e_{1}, L\left(a_{12}\right)\right], b_{12}\right] \\
= & -\left[\left[e_{1}, L\left(a_{12}\right)\right], b_{12}\right] .
\end{aligned}
$$

So, by (18), one achieves

$$
\begin{aligned}
& {\left[L\left(a_{12}\right), b_{12}\right]+\left[a_{12}, L\left(b_{12}\right)\right]} \\
& \quad=\left[e_{2} L\left(a_{12}\right) e_{1}, b_{12}\right]-\left[\left[e_{1}, L\left(a_{12}\right)\right], b_{12}\right] \\
& \quad=2\left[e_{2} L\left(a_{12}\right) e_{1}, b_{12}\right] \in \mathscr{Z}(\mathscr{R}) .
\end{aligned}
$$

It follows from char $\mathscr{R} \neq 2$ that $\left[e_{2} L\left(a_{12}\right) e_{1}, b_{12}\right]=$ $e_{2} L\left(a_{12}\right) e_{1} b_{12}-b_{12} e_{2} L\left(a_{12}\right) e_{1} \in \mathscr{Z}(\mathscr{R})$ for all $b_{12} \in \mathscr{R}_{12}$.
By Lemma 8 , one can get $e_{2} L\left(a_{12}\right) e_{1} b_{12} \in \mathscr{Z}\left(\mathscr{R}_{22}\right)$ for all $b_{12} \in \mathscr{R}_{12}$. It is easily checked that $e_{2} L\left(a_{12}\right) e_{1} \mathscr{R} e_{2}$ is an ideal of $\mathscr{R}_{22}$. So, by the assumption (ii), $e_{2} L\left(a_{12}\right) e_{1} \mathscr{R} e_{2}=\{0\}$, and so $e_{2} L\left(a_{12}\right) e_{1}=0$ by the condition (i). This and (18) imply that $L\left(a_{12}\right)=e_{1} L\left(a_{12}\right) e_{2} \in \mathscr{R}_{12}$, as desired.

Claim 5. For any $a_{i j} \in \mathscr{R}_{i j}$, we have $L\left(-a_{i j}\right)=-L\left(a_{i j}\right), 1 \leq$ $i \neq j \leq 2$.

By (16) and Claims 3 and 4, it is clear that

$$
L\left(-a_{i j}\right)=L\left(\left[\left[a_{i j}, e_{i}\right], e_{j}\right]\right)=\left[\left[L\left(a_{i j}\right), e_{i}\right], e_{j}\right]=-L\left(a_{i j}\right) .
$$

Claim 6. For any $a_{i j} \in \mathscr{R}_{i j}$, we have $L\left(e_{i}+a_{i j}\right)-L\left(a_{i j}\right) \in \mathscr{Z}(\mathscr{R})$, $1 \leq i \neq j \leq 2$.

Let $1 \leq i \neq j \leq 2$ and $a_{i j} \in \mathscr{R}_{i j}$. For any $b_{i j} \in \mathscr{R}_{i j}$, by (16) and Claims 3 and 4 , one has

$$
\begin{aligned}
L\left(b_{i j}\right) & =L\left(\left[\left[e_{i}+a_{i j}, b_{i j}\right], e_{j}\right]\right) \\
& =\left[\left[L\left(e_{i}+a_{i j}\right), b_{i j}\right], e_{j}\right]+L\left(b_{i j}\right) ;
\end{aligned}
$$

that is, $\left[\left[L\left(e_{i}+a_{i j}\right), b_{i j}\right], e_{j}\right]=0$. Note that $\left[\left[L\left(a_{i j}\right), b_{i j}\right], e_{j}\right]=0$ by Claim 4 . So

$$
\left[\left[L\left(e_{i}+a_{i j}\right)-L\left(a_{i j}\right), b_{i j}\right], e_{j}\right]=0 .
$$

Similarly, for any $b_{j i} \in \mathscr{R}_{j i}$, by the relations $-L\left(b_{j i}\right)=$ $L\left(-b_{j i}\right)=L\left(\left[\left[e_{i}+a_{i j}, b_{j i}\right], e_{i}\right]\right)$ and $\left[\left[L\left(a_{i j}\right), b_{j i}\right], e_{i}\right]=0$, one can check

$$
\left[\left[L\left(e_{i}+a_{i j}\right)-L\left(a_{i j}\right), b_{j i}\right], e_{i}\right]=0
$$

Combining (24) and (25) and by Lemma 10, we achieve

$$
\begin{aligned}
& e_{1}\left(L\left(e_{i}+a_{i j}\right)-L\left(a_{i j}\right)\right) e_{1} \\
& \quad+e_{2}\left(L\left(e_{i}+a_{i j}\right)-L\left(a_{i j}\right)\right) e_{2} \in \mathscr{Z}(\mathscr{R}) .
\end{aligned}
$$

Thus, to complete the proof of the claim, we still need to check $e_{1}\left(L\left(e_{i}+a_{i j}\right)-L\left(a_{i j}\right)\right) e_{2}=e_{2}\left(L\left(e_{i}+a_{i j}\right)-L\left(a_{i j}\right)\right) e_{1}=0$. In fact, by (16), we have

$$
\begin{aligned}
& L\left(a_{i j}\right)=L\left(\left[\left[e_{i}+a_{i j}, e_{1}\right], e_{1}\right]\right)=\left[\left[L\left(e_{i}+a_{i j}\right), e_{1}\right], e_{1}\right] \\
& L\left(a_{i j}\right)=L\left(\left[\left[a_{i j}, e_{1}\right], e_{1}\right]\right)=\left[\left[L\left(a_{i j}\right), e_{1}\right], e_{1}\right] .
\end{aligned}
$$

It follows that $\left[\left[L\left(e_{i}+a_{i j}\right)-L\left(a_{i j}\right), e_{1}\right], e_{1}\right]=0$, which implies $e_{1}\left(L\left(e_{i}+a_{i j}\right)-L\left(a_{i j}\right)\right) e_{2}=e_{2}\left(L\left(e_{i}+a_{i j}\right)-L\left(a_{i j}\right)\right) e_{1}=0$.

Claim 7. $L$ is additive on $\mathscr{R}_{i j}, 1 \leq i \neq j \leq 2$. 
Take any $a_{i j}, b_{i j} \in \mathscr{R}_{i j}(1 \leq i \neq j \leq 2)$. By (16) and Claims 3-6, one obtains

$$
\begin{aligned}
L\left(a_{i j}+b_{i j}\right)= & L\left(\left[\left[e_{i}-a_{i j}, e_{i}+b_{i j}\right], e_{j}\right]\right) \\
= & {\left[\left[L\left(e_{i}-a_{i j}\right), e_{i}+b_{i j}\right], e_{j}\right] } \\
& +\left[\left[e_{i}-a_{i j}, L\left(e_{i}+b_{i j}\right)\right], e_{j}\right] \\
= & {\left[\left[-L\left(a_{i j}\right), e_{i}+b_{i j}\right], e_{j}\right] } \\
& +\left[\left[e_{i}-a_{i j}, L\left(b_{i j}\right)\right], e_{j}\right] \\
= & L\left(a_{i j}\right)+L\left(b_{i j}\right) .
\end{aligned}
$$

Claim 8. For $1 \leq i \neq j \leq 2$, we have $L\left(\mathscr{R}_{i i}\right) \subseteq\left(\mathscr{R}_{i i}+\mathscr{R}_{j j}\right)$, $L\left(\mathscr{R}_{i i}+\mathscr{R}_{i i}\right) \subseteq\left(\mathscr{R}_{i i}+\mathscr{R}_{j j}\right)$, and $L\left(\mathscr{R}_{i i}+\mathscr{R}_{j j}\right) \subseteq\left(\mathscr{R}_{i i}+\mathscr{R}_{j j}\right)$.

Let $1 \leq i \neq j \leq 2$. For any $a_{i i}, b_{i i} \in \mathscr{R}_{i i}$ and $a_{j j} \in \mathscr{R}_{j j}$, by (16) and Claim 3, one has

$$
\begin{aligned}
& 0=L\left(\left[\left[a_{i i}+a_{j j}, e_{j}\right], e_{j}\right]\right)=\left[\left[L\left(a_{i i}+a_{j j}\right), e_{j}\right], e_{j}\right], \\
& 0=L\left(\left[\left[a_{i i}, e_{j}\right], e_{j}\right]\right)=\left[\left[L\left(a_{i i}\right), e_{j}\right], e_{j}\right], \\
& 0=L\left(\left[\left[a_{i i}+b_{i i}, e_{j}\right], e_{j}\right]\right)=\left[\left[L\left(a_{i i}+b_{i i}\right), e_{j}\right], e_{j}\right] .
\end{aligned}
$$

A simple calculation yields $e_{1} L\left(a_{i i}+a_{j j}\right) e_{2}=e_{2} L\left(a_{i i}+a_{j j}\right) e_{1}=$ $0, e_{1} L\left(a_{i i}\right) e_{2}=e_{2} L\left(a_{i i}\right) e_{1}=0$, and $e_{1} L\left(a_{i i}+b_{i i}\right) e_{2}=e_{2} L\left(a_{i i}+\right.$ $\left.b_{i i}\right) e_{1}=0$. So the claim is true.

Claim 9. For any $a_{11} \in \mathscr{R}_{11}$ and $a_{22} \in \mathscr{R}_{22}$, we have $L\left(a_{11}+\right.$ $\left.a_{22}\right)-L\left(a_{11}\right)-L\left(a_{22}\right) \in \mathscr{Z}(\mathscr{R})$.

Take any $a_{11} \in \mathscr{R}_{11}$ and $a_{22} \in \mathscr{R}_{22}$ and write $L\left(a_{11}+a_{22}\right)-$ $L\left(a_{11}\right)-L\left(a_{22}\right)=s_{11}+s_{12}+s_{21}+s_{22}$. Note that, by Claim 8 , one has $L\left(a_{11}+a_{22}\right)-L\left(a_{11}\right)-L\left(a_{22}\right)=s_{11}+s_{22}$. So, to prove the claim, one only needs to check $s_{11}+s_{22} \in \mathscr{Z}(\mathscr{R})$. have

To do this, taking any $b_{12} \in \mathscr{R}_{12}$, by Claims $3-5$ and 7, we

$$
\begin{aligned}
L\left(\left[a_{11}+a_{22}, b_{12}\right]\right)= & L\left(a_{11} b_{12}-b_{12} a_{22}\right) \\
= & L\left(a_{11} b_{12}\right)-L\left(b_{12} a_{22}\right) \\
= & L\left(\left[\left[a_{11}, b_{12}\right], e_{2}\right]\right)-L\left(\left[\left[b_{12}, a_{22}\right], e_{2}\right]\right) \\
= & {\left[\left[L\left(a_{11}\right), b_{12}\right], e_{2}\right]+\left[\left[a_{11}, L\left(b_{12}\right)\right], e_{2}\right] } \\
& -\left[\left[L\left(b_{12}\right), a_{22}\right], e_{2}\right] \\
& -\left[\left[b_{12}, L\left(a_{22}\right)\right], e_{2}\right] \\
= & {\left[\left[L\left(a_{11}\right), b_{12}\right], e_{2}\right]+\left[a_{11}, L\left(b_{12}\right)\right] } \\
& +\left[a_{22}, L\left(b_{12}\right)\right]+\left[\left[L\left(a_{22}\right), b_{12}\right], e_{2}\right],
\end{aligned}
$$

$$
\begin{aligned}
L\left(\left[a_{11}+a_{22}, b_{12}\right]\right)= & L\left(\left[\left[a_{11}+a_{22}, b_{12}\right], e_{2}\right]\right) \\
= & {\left[\left[L\left(a_{11}+a_{22}\right), b_{12}\right], e_{2}\right] } \\
& +\left[\left[a_{11}+a_{22}, L\left(b_{12}\right)\right], e_{2}\right] \\
= & {\left[\left[L\left(a_{11}+a_{22}\right), b_{12}\right], e_{2}\right] } \\
& +\left[a_{11}, L\left(b_{12}\right)\right]+\left[a_{22}, L\left(b_{12}\right)\right] .
\end{aligned}
$$

Combining the above two equations yields

$$
\begin{array}{r}
{\left[\left[L\left(a_{11}+a_{22}\right)-L\left(a_{11}\right)-L\left(a_{22}\right), b_{12}\right], e_{2}\right]=0} \\
\forall b_{12} \in \mathscr{R}_{12} .
\end{array}
$$

Similar to the above discussion, one can also prove that

$$
\begin{array}{r}
{\left[\left[L\left(a_{11}+a_{22}\right)-L\left(a_{11}\right)-L\left(a_{22}\right), b_{21}\right], e_{1}\right]=0} \\
\forall b_{21} \in \mathscr{R}_{21} .
\end{array}
$$

Now, by Lemma 10 , it follows that $s_{11}+s_{22} \in \mathscr{Z}(\mathscr{R})$, and the claim holds.

Claim 10. For any $a \in \mathscr{R}$, any $a_{12} \in \mathscr{R}_{12}$, and any $a_{21} \in \mathscr{R}_{21}$, we have

(i) $L\left(a+a_{12}\right)-L(a)-e_{1}\left(L\left(a+a_{12}\right)-L(a)\right) e_{2} \in \mathscr{Z}(\mathscr{R})$;

(ii) $L\left(a+a_{21}\right)-L(a)-e_{2}\left(L\left(a+a_{21}\right)-L(a)\right) e_{1} \in \mathscr{Z}(\mathscr{R})$.

For any $a, c \in \mathscr{R}$ and any $a_{12}, b_{12} \in \mathscr{R}_{12}$, since $[[a+$ $\left.\left.a_{12}, b_{12}\right], c\right]=\left[\left[a, b_{12}\right], c\right]$, by Claim 4 , one can easily get $\left[\left[L\left(a+a_{12}\right), b_{12}\right], c\right]=\left[\left[L(a), b_{12}\right], c\right]$; that is,

$$
\left[\left[L\left(a+a_{12}\right)-L(a), b_{12}\right], c\right]=0 \quad \forall c \in \mathscr{R} .
$$

Write $t=L\left(a+a_{12}\right)-L(a)=t_{11}+t_{12}+t_{21}+t_{22}$. Then (33) implies that

$$
\left[t, b_{12}\right]=t_{11} b_{12}+t_{21} b_{12}-b_{12} t_{21}-b_{12} t_{22} \in \mathscr{Z}(\mathscr{R}) .
$$

Letting $c=e_{2}$ in (33), a simple calculation yields

$$
t_{11} b_{12}=b_{12} t_{22} \quad \forall b_{12} \in \mathscr{R}_{12}
$$

So (34) is reduced to $t_{21} b_{12}-b_{12} t_{21} \in \mathscr{Z}(\mathscr{R})$, which and Lemma 8 imply that $t_{21} b_{12} \in \mathscr{Z}\left(\mathscr{R}_{22}\right)$ holds for all $b_{12} \in \mathscr{R}_{12}$. Note that $t_{21} e_{1} \mathscr{R} e_{2}$ is an ideal of $\mathscr{R}_{22}$. It follows from the assumption (ii) that $t_{21} e_{1} \mathscr{R} e_{2}=\{0\}$, and so

$$
t_{21}=0 \text {. }
$$

On the other hand, by using the equation $\left[\left[a+a_{12}, b_{21}\right], e_{2}\right]=$ $\left[\left[a, b_{21}\right], e_{2}\right]$, one can also show

$$
t_{22} b_{21}=b_{21} t_{11} \quad \forall b_{21} \in \mathscr{R}_{21}, t_{12}=0 .
$$

Combining (35)-(37) and Lemma 8, we get that (i) holds.

The proof of (ii) is similar and we omit it here. 
Claim 11. For any $a_{i j} \in \mathscr{R}_{i j}(1 \leq i, j \leq 2)$, we have

(i) $L\left(a_{12}\right)=e_{1} L\left(a_{11}+a_{12}+a_{22}\right) e_{2}$ and $e_{2} L\left(a_{11}+a_{12}+\right.$ $\left.a_{22}\right) e_{1}=0$

(ii) $L\left(a_{21}\right)=e_{2} L\left(a_{11}+a_{21}+a_{22}\right) e_{1}$ and $e_{1} L\left(a_{11}+a_{21}+\right.$ $\left.a_{22}\right) e_{2}=0$.

Here, we only give the proof of (i). The proof of (ii) is similar.

For any $a_{i j} \in \mathscr{R}_{i j}(1 \leq i, j \leq 2)$, by Claim 3 , one gets

$$
\begin{aligned}
L\left(a_{12}\right)= & L\left(\left[\left[a_{11}+a_{12}+a_{22}, e_{2}\right], e_{2}\right]\right) \\
= & {\left[\left[L\left(a_{11}+a_{12}+a_{22}\right), e_{2}\right], e_{2}\right] } \\
= & e_{1} L\left(a_{11}+a_{12}+a_{22}\right) e_{2} \\
& +e_{2} L\left(a_{11}+a_{12}+a_{22}\right) e_{1},
\end{aligned}
$$

which, together with Claim 4 , implies $L\left(a_{12}\right)=e_{1} L\left(a_{11}+a_{12}+\right.$ $\left.a_{22}\right) e_{2}$ and $e_{2} L\left(a_{11}+a_{12}+a_{22}\right) e_{1}=0$, as desired.

Claim 12. For any $a_{i j} \in \mathscr{R}_{i j}(1 \leq i, j \leq 2)$, we have

(i) $L\left(a_{11}+a_{12}+a_{22}\right)-L\left(a_{11}\right)-L\left(a_{12}\right)-L\left(a_{22}\right) \in \mathscr{Z}(\mathscr{R})$;

(ii) $L\left(a_{11}+a_{21}+a_{22}\right)-L\left(a_{11}\right)-L\left(a_{21}\right)-L\left(a_{22}\right) \in \mathscr{Z}(\mathscr{R})$.

Still, we only give the proof of (i).

Take any $a_{11} \in \mathscr{R}_{11}, a_{12} \in \mathscr{R}_{12}$, and $a_{22} \in \mathscr{R}_{22}$. Firstly, by Claims 8, 9, and 11, there exists some $z \in \mathscr{Z}(\mathscr{R})$ such that

$$
\begin{aligned}
& L\left(a_{11}+a_{12}+a_{22}\right)-L\left(a_{11}\right)-L\left(a_{12}\right)-L\left(a_{22}\right) \\
& =L\left(a_{11}+a_{12}+a_{22}\right)-L\left(a_{11}+a_{22}\right)-L\left(a_{12}\right)+z \\
& =e_{1}\left(L\left(a_{11}+a_{12}+a_{22}\right)-L\left(a_{11}+a_{22}\right)\right) e_{1} \\
& \quad+e_{2}\left(L\left(a_{11}+a_{12}+a_{22}\right)-L\left(a_{11}+a_{22}\right)\right) e_{2}+z .
\end{aligned}
$$

Next, for any $b_{12} \in \mathscr{R}_{12}$, by Claims 3 and 4 , one has

$$
\begin{aligned}
L( & \left.\left(\left[a_{11}+a_{12}+a_{22}, b_{12}\right], e_{2}\right]\right) \\
= & {\left[\left[L\left(a_{11}+a_{12}+a_{22}\right), b_{12}\right], e_{2}\right] } \\
& +\left[\left[a_{11}+a_{12}+a_{22}, L\left(b_{12}\right)\right], e_{2}\right] \\
= & {\left[\left[L\left(a_{11}+a_{12}+a_{22}\right), b_{12}\right], e_{2}\right] } \\
& +\left[\left[a_{11}+a_{22}, L\left(b_{12}\right)\right], e_{2}\right], \\
L([[ & \left.\left.\left.a_{11}+a_{12}+a_{22}, b_{12}\right], e_{2}\right]\right) \\
= & L\left(\left[\left[a_{11}+a_{22}, b_{12}\right], e_{2}\right]\right) \\
= & {\left[\left[L\left(a_{11}+a_{22}\right), b_{12}\right], e_{2}\right] } \\
& +\left[\left[a_{11}+a_{22}, L\left(b_{12}\right)\right], e_{2}\right] .
\end{aligned}
$$

Comparing the above equations, we get

$$
\left[\left[L\left(a_{11}+a_{12}+a_{22}\right)-L\left(a_{11}+a_{22}\right), b_{12}\right], e_{2}\right]=0 .
$$

Finally, for any $b_{21} \in \mathscr{R}_{21}$, a similar argument to the above achieves

$$
\left[\left[L\left(a_{11}+a_{12}+a_{22}\right)-L\left(a_{11}+a_{22}\right), b_{21}\right], e_{1}\right]=0 .
$$
holds.

Now, combining (39)-(42) and Lemma 10, the claim

Claim 13. For any $a_{i j} \in \mathscr{R}_{i j}(1 \leq i, j \leq 2)$, we have $L\left(a_{11}+\right.$ $\left.a_{12}+a_{21}+a_{22}\right)-L\left(a_{11}\right)-L\left(a_{12}\right)-L\left(a_{21}\right)-L\left(a_{22}\right) \in \mathscr{Z}(\mathscr{R})$.

Take any $a_{i j} \in \mathscr{R}_{i j}(1 \leq i, j \leq 2)$ and write

$$
\begin{aligned}
t= & L\left(a_{11}+a_{12}+a_{21}+a_{22}\right)-L\left(a_{11}\right) \\
& -L\left(a_{12}\right)-L\left(a_{21}\right)-L\left(a_{22}\right) .
\end{aligned}
$$

For any $b_{12} \in \mathscr{R}_{12}$ and any $b_{21} \in \mathscr{R}_{21}$, by Claims 4,10 , and 12 , one has

$$
\begin{aligned}
{\left[t, b_{12}\right]=} & {\left[L\left(a_{11}+a_{12}+a_{21}+a_{22}\right)\right.} \\
& \left.-L\left(a_{11}\right)-L\left(a_{21}\right)-L\left(a_{22}\right), b_{12}\right] \\
= & {\left[L\left(a_{11}+a_{12}+a_{21}+a_{22}\right)\right.} \\
& \left.-L\left(a_{11}+a_{21}+a_{22}\right), b_{12}\right]=0, \\
{\left[t, b_{21}\right]=\left[L\left(a_{11}+a_{12}+a_{21}+a_{22}\right)-L\left(a_{11}\right)\right.} & \left.-L\left(a_{12}\right)-L\left(a_{22}\right), b_{21}\right] \\
= & {\left[L\left(a_{11}+a_{12}+a_{21}+a_{22}\right)\right.} \\
& \left.-L\left(a_{11}+a_{12}+a_{22}\right), b_{21}\right]=0 .
\end{aligned}
$$

Now the above two equations and Lemma 9 imply $t \in \mathscr{Z}(\mathscr{R})$.

Claim 14. For any $a_{i i}, b_{i i} \in \mathscr{R}_{i i}$, we have $L\left(a_{i i}+b_{i i}\right)-L\left(a_{i i}\right)-$ $L\left(b_{i i}\right) \in \mathscr{Z}(\mathscr{R}), i=1,2$.

Let $1 \leq i \neq j \leq 2$ and $a_{i i}, b_{i i} \in \mathscr{R}_{i i}$ be arbitrary. For any $c_{i j} \in \mathscr{R}_{i j}$, by (16) and Claims 3 and 4 , we have

$$
\begin{aligned}
L\left(\left[a_{i i}+b_{i i}, c_{i j}\right]\right)= & L\left(\left[a_{i i}, c_{i j}\right]+\left[b_{i i}, c_{i j}\right]\right) \\
= & L\left(\left[a_{i i}, c_{i j}\right]\right)+L\left(\left[b_{i i}, c_{i j}\right]\right) \\
= & L\left(\left[\left[a_{i i}, c_{i j}\right], e_{j}\right]\right)+L\left(\left[\left[b_{i i}, c_{i j}\right], e_{j}\right]\right) \\
= & {\left[\left[L\left(a_{i i}\right), c_{i j}\right], e_{j}\right]+\left[\left[a_{i i}, L\left(c_{i j}\right)\right], e_{j}\right] } \\
& +\left[\left[L\left(b_{i i}\right), c_{i j}\right], e_{j}\right]+\left[\left[b_{i i}, L\left(c_{i j}\right)\right], e_{j}\right], \\
L\left(\left[a_{i i}+b_{i i}, c_{i j}\right]\right)= & L\left(\left[\left[a_{i i}+b_{i i}, c_{i j}\right], e_{j}\right]\right) \\
= & {\left[\left[L\left(a_{i i}+b_{i i}\right), c_{i j}\right], e_{j}\right] } \\
& +\left[\left[a_{i i}+b_{i i}, L\left(c_{i j}\right)\right], e_{j}\right] .
\end{aligned}
$$

It follows that

$$
\begin{array}{r}
{\left[\left[L\left(a_{i i}+b_{i i}\right)-L\left(a_{i i}\right)-L\left(b_{i i}\right), c_{i j}\right], e_{j}\right]=0} \\
\forall c_{i j} \in \mathscr{R}_{i j} .
\end{array}
$$


Similarly, one can check

$$
\begin{array}{r}
{\left[\left[L\left(a_{i i}+b_{i i}\right)-L\left(a_{i i}\right)-L\left(b_{i i}\right), c_{j i}\right], e_{i}\right]=0} \\
\forall c_{j i} \in \mathscr{R}_{j i} .
\end{array}
$$

Now, together with Lemma 10 and Claim 8, (46)-(47) imply that the claim holds.

Claim 15. $L(a+b)-L(a)-L(b) \in \mathscr{Z}(\mathscr{R})$ for all $a, b \in \mathscr{R}$. Therefore, Theorem 1 holds.

In fact, by Claim 7 and Claims 13 and 14, it is easily seen that the claim is true.

The proof of the theorem is complete.

Proof of Theorem 2. The "if" part is clear. We will prove the "only if" part by several claims.

Claim 1. For any $a_{i i} \in \mathscr{R}_{i i}$, there exists a map $f_{i}: \mathscr{R}_{i i} \rightarrow$ $\mathscr{Z}(\mathscr{R})$ such that $L\left(a_{i i}\right)-f_{i}\left(a_{i i}\right) \in \mathscr{R}_{i i}, i=1,2$.

We only give the proof for $a_{11}$ here. The proof for any $a_{22}$ is similar.

For any $a_{11} \in \mathscr{R}_{11}$, by Claim 8 in the proof of Theorem 1 , we have

$$
L\left(a_{11}\right)=e_{1} L\left(a_{11}\right) e_{1}+e_{2} L\left(a_{11}\right) e_{2} \in \mathscr{R}_{11}+\mathscr{R}_{22} .
$$

Now, taking any $b_{22} \in \mathscr{R}_{22}$ and any $c \in \mathscr{R}$, one has

$$
\begin{aligned}
0 & =L\left(\left[\left[a_{11}, b_{22}\right], c\right]\right) \\
& =\left[\left[L\left(a_{11}\right), b_{22}\right], c\right]+\left[\left[a_{11}, L\left(b_{22}\right)\right], c\right],
\end{aligned}
$$

which implies $\left[L\left(a_{11}\right), b_{22}\right]+\left[a_{11}, L\left(b_{22}\right)\right] \in \mathscr{Z}(\mathscr{R})$. It follows that $\left[L\left(a_{11}\right), b_{22}\right] \in \mathscr{Z}\left(\mathscr{R}_{22}\right)$. By the assumption (iii) in the theorem, one obtains $\left[L\left(a_{11}\right), b_{22}\right]=0$ for all $b_{22} \in \mathscr{R}_{22}$; that is, $e_{2} L\left(a_{11}\right) e_{2} b e_{2}=e_{2} b e_{2} L\left(a_{11}\right) e_{2}$ for all $b \in \mathscr{R}$. It follows from Lemma 12 that $e_{2} L\left(a_{11}\right) e_{2}=f_{1}\left(a_{11}\right) e_{2}$ for some $f_{1}\left(a_{11}\right) \in$ $\mathscr{Z}(\mathscr{R})$. Hence

$$
\begin{aligned}
L\left(a_{11}\right)= & e_{1} L\left(a_{11}\right) e_{1}+f_{1}\left(a_{11}\right) e_{2} \\
= & e_{1} L\left(a_{11}\right) e_{1}-f_{1}\left(a_{11}\right) e_{1} \\
& +f_{1}\left(a_{11}\right) \in \mathscr{R}_{11}+\mathscr{Z}(\mathscr{R}) .
\end{aligned}
$$

The claim holds.

Now define two maps $\delta: \mathscr{R} \rightarrow \mathscr{R}$ and $f: \mathscr{R} \rightarrow \mathscr{Z}(\mathscr{R})$, respectively, by

$$
\begin{aligned}
\delta(a)= & L\left(a_{11}\right)+L\left(a_{12}\right)+L\left(a_{21}\right) \\
+ & L\left(a_{22}\right)-f_{1}\left(a_{11}\right)-f_{2}\left(a_{22}\right), \\
& f(a)=L(a)-\delta(a)
\end{aligned}
$$

for all $a=a_{11}+a_{12}+a_{21}+a_{22} \in \mathscr{R}$. Then, by Claim 4 in the proof of Theorem 1 and Claim 1, we have that

$$
\delta\left(\mathscr{R}_{i j}\right)=L\left(\mathscr{R}_{i j}\right) \subseteq \mathscr{R}_{i j}, \quad \delta\left(\mathscr{R}_{i i}\right) \subseteq \mathscr{R}_{i i}, \quad 1 \leq i \neq j \leq 2 .
$$

Moreover, $\delta\left(e_{i}\right) \in \mathscr{Z}(\mathscr{R})$ for $i=1,2$.

Claim 2. $\delta$ is additive on $\mathscr{R}$.
By Claim 7 in the proof of Theorem $1, \delta$ is additive on $\mathscr{R}_{12}$ and $\mathscr{R}_{21}$.

Let $i \in\{1,2\}$. For any $a_{i i}, b_{i i} \in \mathscr{R}_{i i}$, by Claim 14 in the proof of Theorem 1, (52), and the definition of $\delta$, we have

$$
\begin{aligned}
\delta\left(a_{i i}\right. & \left.+b_{i i}\right)-\delta\left(a_{i i}\right)-\delta\left(b_{i i}\right) \\
= & L\left(a_{i i}+b_{i i}\right)-L\left(a_{i i}\right)-L\left(b_{i i}\right)-f\left(a_{i i}+b_{i i}\right) \\
& +f\left(a_{i i}\right)+f\left(b_{i i}\right) \in \mathscr{Z}(\mathscr{R}) \cap \mathscr{R}_{i i} .
\end{aligned}
$$

It follows from Lemma 11 that $\delta\left(a_{i i}+b_{i i}\right)-\delta\left(a_{i i}\right)-\delta\left(b_{i i}\right)=0$; that is, $\delta$ is additive on $\mathscr{R}_{i i}, i=1,2$.

Now, for any $a=\sum_{i, j=1}^{2} a_{i j}, b=\sum_{i, j=1}^{2} b_{i j} \in \mathscr{R}$, by what the above proved, one gets

$$
\begin{aligned}
\delta(a+b)= & \delta\left(\sum_{i, j}^{2}\left(a_{i j}+b_{i j}\right)\right) \\
= & L\left(a_{11}+b_{11}\right)+L\left(a_{12}+b_{12}\right) \\
& +L\left(a_{21}+b_{21}\right)+L\left(a_{22}+b_{22}\right) \\
& -f_{1}\left(a_{11}+b_{11}\right)-f_{2}\left(a_{22}+b_{22}\right) \\
= & \delta\left(a_{11}+b_{11}\right)+\delta\left(a_{12}+b_{12}\right) \\
& +\delta\left(a_{21}+b_{21}\right)+\delta\left(a_{22}+b_{22}\right) \\
= & \sum_{i, j=1}^{2} \delta\left(a_{i j}\right)+\sum_{i, j=1}^{2} \delta\left(b_{i j}\right)=\delta(a)+\delta(b) .
\end{aligned}
$$

Hence $\delta$ is additive on $\mathscr{R}$.

Claim 3. $\delta$ is a derivation.

We will divide the proof of the claim into five steps.

Step 1. For any $a_{i i} \in \mathscr{R}_{i i}$ and any $b_{i j} \in \mathscr{R}_{i j}$, we have $\delta\left(a_{i i} b_{i j}\right)=$ $\delta\left(a_{i i}\right) b_{i j}+a_{i i} \delta\left(b_{i j}\right), 1 \leq i \neq j \leq 2$.

For any $a_{i i} \in \mathscr{R}_{i i}$ and $b_{i j} \in \mathscr{R}_{i j}(1 \leq i \neq j \leq 2)$, by the definition of $\delta$ and (33), one obtains

$$
\begin{aligned}
\delta\left(a_{i i} b_{i j}\right) & =L\left(a_{i i} b_{i j}\right)=L\left(\left[\left[a_{i i}, b_{i j}\right], e_{j}\right]\right) \\
& =\left[\left[L\left(a_{i i}\right), b_{i j}\right], e_{j}\right]+\left[\left[a_{i i}, L\left(b_{i j}\right)\right], e_{j}\right] \\
& =\left[\left[\delta\left(a_{i i}\right), b_{i j}\right], e_{j}\right]+\left[\left[a_{i i}, \delta\left(b_{i j}\right)\right], e_{j}\right] \\
& =\delta\left(a_{i i}\right) b_{i j}+a_{i i} \delta\left(b_{i j}\right) .
\end{aligned}
$$

By a similar argument to that of Step 1, one can show the following.

Step 2. For any $a_{i j} \in \mathscr{R}_{i j}$ and any $b_{j j} \in \mathscr{R}_{j j}$, we have $\delta\left(a_{i j} b_{j j}\right)=$ $\delta\left(a_{i j}\right) b_{j j}+a_{i j} \delta\left(b_{j j}\right), 1 \leq i \neq j \leq 2$.

Step 3. For any $a_{i i}, b_{i i} \in \mathscr{R}_{i i}$, we have $\delta\left(a_{i i} b_{i i}\right)=\delta\left(a_{i i}\right) b_{i i}+$ $a_{i i} \delta\left(b_{i i}\right), i=1,2$. 
Take any $a_{i i}, b_{i i} \in \mathscr{R}_{i i}$ and any $c_{i j} \in \mathscr{R}_{i j}(1 \leq i \neq j \leq 2)$. By Step 1 , one has

$$
\begin{aligned}
\delta\left(a_{i i} b_{i i} c_{i j}\right)= & \delta\left(a_{i i}\right) b_{i i} c_{i j}+a_{i i} \delta\left(b_{i i} c_{i j}\right) \\
= & \delta\left(a_{i i}\right) b_{i i} c_{i j}+a_{i i} \delta\left(b_{i i}\right) c_{i j} \\
& +a_{i i} b_{i i} \delta\left(c_{i j}\right), \\
\delta\left(a_{i i} b_{i i} c_{i j}\right)= & \delta\left(a_{i i} b_{i i}\right) c_{i j}+a_{i i} b_{i i} \delta\left(c_{i j}\right) .
\end{aligned}
$$

The above two equations yield $\left(\delta\left(a_{i i} b_{i i}\right)-\delta\left(a_{i i}\right) b_{i i}-\right.$ $\left.a_{i i} \delta\left(b_{i i}\right)\right) c_{i j}=0$; that is, $\left(\delta\left(a_{i i} b_{i i}\right)-\delta\left(a_{i i}\right) b_{i i}-a_{i i} \delta\left(b_{i i}\right)\right) e_{i} c e_{j}=0$ for all $c \in \mathscr{R}$. Note that $\delta\left(a_{i i} b_{i i}\right)-\delta\left(a_{i i}\right) b_{i i}-a_{i i} \delta\left(b_{i i}\right) \in \mathscr{R}_{i i}$. It follows that $\delta\left(a_{i i} b_{i i}\right)-\delta\left(a_{i i}\right) b_{i i}-a_{i i} \delta\left(b_{i i}\right)=0$.

Step 4. For any $a_{i j} \in \mathscr{R}_{i j}$ and any $b_{j i} \in \mathscr{R}_{j i}$, we have $\delta\left(a_{i j} b_{j i}\right)=$ $\delta\left(a_{i j}\right) b_{j i}+a_{i j} \delta\left(b_{j i}\right), 1 \leq i \neq j \leq 2$.

For any $a_{i j} \in \mathscr{R}_{i j}$ and any $b_{j i} \in \mathscr{R}_{j i}(1 \leq i \neq j \leq 2)$, noting that $L\left(e_{j}\right) \in \mathscr{E}(\mathscr{R})$, by (52), Claim 2 , and the definitions of $\delta$ and $f$, we have

$$
\begin{aligned}
& \delta\left(a_{i j} b_{j i}\right)-\delta\left(b_{j i} a_{i j}\right) \\
&= \delta\left(a_{i j} b_{j i}-b_{j i} a_{i j}\right) \\
&= L\left(a_{i j} b_{j i}-b_{j i} a_{i j}\right)-f\left(a_{i j} b_{j i}-b_{j i} a_{i j}\right) \\
&= L\left(\left[\left[a_{i j}, e_{j}\right], b_{j i}\right]\right)-f\left(a_{i j} b_{j i}-b_{j i} a_{i j}\right) \\
&= {\left[\left[L\left(a_{i j}\right), e_{j}\right], b_{j i}\right]+\left[\left[a_{i j}, e_{j}\right], L\left(b_{j i}\right)\right] } \\
&-f\left(a_{i j} b_{j i}-b_{j i} a_{i j}\right) \\
&= {\left[\delta\left(a_{i j}\right), b_{j i}\right]+\left[a_{i j}, \delta\left(b_{j i}\right)\right]-f\left(a_{i j} b_{j i}-b_{j i} a_{i j}\right) } \\
&= \delta\left(a_{i j}\right) b_{j i}+a_{i j} \delta\left(b_{j i}\right)-b_{j i} \delta\left(a_{i j}\right) \\
&-\delta\left(b_{j i}\right) a_{i j}-f\left(a_{i j} b_{j i}-b_{j i} a_{i j}\right) .
\end{aligned}
$$

Multiplying $e_{i}$ from both sides in the above equation, and noting that (52), one obtains

$$
\delta\left(a_{i j} b_{j i}\right)=\delta\left(a_{i j}\right) b_{j i}+a_{i j} \delta\left(b_{j i}\right)-f\left(a_{i j} b_{j i}-b_{j i} a_{i j}\right) e_{i} .
$$

Define a set

$$
\begin{gathered}
\mathcal{U}_{i}=\left\{\delta\left(a_{i j} b_{j i}\right)-\delta\left(a_{i j}\right) b_{j i}-a_{i j} \delta\left(b_{j i}\right): a_{i j} \in \mathscr{R}_{i j},\right. \\
\left.b_{j i} \in \mathscr{R}_{j i}, 1 \leq i \neq j \leq 2\right\} .
\end{gathered}
$$

It is easily seen that $\mathscr{U}_{i} \subseteq \mathscr{Z}(\mathscr{R}) e_{i}=\mathscr{Z}\left(\mathscr{R}_{i i}\right)$. Also note that, for any $c_{i i} \in \mathscr{R}_{i i}$,

$$
\begin{aligned}
c_{i i} & \left(\delta\left(a_{i j} b_{j i}\right)-\delta\left(a_{i j}\right) b_{j i}-a_{i j} \delta\left(b_{j i}\right)\right) \\
= & c_{i i} \delta\left(a_{i j} b_{j i}\right)+\delta\left(c_{i i}\right) a_{i j} b_{j i}-\delta\left(c_{i i}\right) a_{i j} b_{j i} \\
& -c_{i i} \delta\left(a_{i j}\right) b_{j i}-c_{i i} a_{i j} \delta\left(b_{j i}\right) \\
= & \delta\left(c_{i i} a_{i j} b_{j i}\right)-\delta\left(c_{i i} a_{i j}\right) b_{j i}-c_{i i} a_{i j} \delta\left(b_{j i}\right) \in \mathcal{U}_{i} .
\end{aligned}
$$

Thus, $\mathscr{U}_{i}$ is a central ideal of $\mathscr{R}_{i i}$. It follows from the assumption (ii) that $\mathscr{U}_{i}=\{0\}$. So $\delta\left(a_{i j} b_{j i}\right)=\delta\left(a_{i j}\right) b_{j i}+a_{i j} \delta\left(b_{j i}\right)$ for all $a_{i j} \in \mathscr{R}_{i j}$ and $b_{j i} \in \mathscr{R}_{j i}, 1 \leq i \neq j \leq 2$.

Step 5. For any $a, b \in \mathscr{R}$, we have $\delta(a b)=\delta(a) b+a \delta(b)$; that is, $\delta$ is a derivation.

By Claim 2 and Steps 1-4, it is easily checked that the step is true.

Claim 4. $f$ satisfies $f([[a, b], c])=0$ for all $a, b, c \in \mathscr{R}$.

In fact, for any $a, b, c \in \mathscr{R}$, by the definition of $f$ and Claim 3, we have

$$
\begin{aligned}
f([[a, b], c])= & L([[a, b], c])-\delta([[a, b], c]) \\
= & {[[L(a), b], c]+[[a, L(b)], c]+[[a, b], L(c)] } \\
& -[[\delta(a), b], c]-[[a, \delta(b)], c] \\
& -[[a, b], \delta(c)] \\
= & {[[f(a), b], c]+[[a, f(b)], c]+[[a, b], f(c)] } \\
= & 0 .
\end{aligned}
$$

The proof of the theorem is complete.

\section{Conflict of Interests}

The author declares that there is no conflict of interests regarding the publication of this paper.

\section{Acknowledgments}

The author wishes to express her thanks to the referees for their helpful comments and suggestions. This work is partially supported by National Natural Science Foundation of China (11101250) and Youth Foundation of Shanxi Province (2012021004).

\section{References}

[1] M. Brešar, "Commuting traces of biadditive mappings, commutativity-preserving mappings and Lie mappings," Transactions of the American Mathematical Society, vol. 335, no. 2, pp. 525-546, 1993.

[2] M. Mathieu and A. R. Villena, "The structure of Lie derivations on C*-algebras," Journal of Functional Analysis, vol. 202, no. 2, pp. 504-525, 2003.

[3] X. F. Qi and J. C. Hou, "Additive Lie ( $\xi$-Lie) derivations and generalized Lie ( $\xi$-Lie) derivations on nest algebras," Linear Algebra and its Applications, vol. 431, no. 5-7, pp. 843-854, 2009.

[4] C. R. Miers, "Lie triple derivations of von Neumann algebras," Proceedings of the American Mathematical Society, vol. 71, no. 1, pp. 57-61, 1978.

[5] T. Wang and F. Lu, "Lie triple derivations on $\mathscr{J}$-subspace lattice algebras," Abstract and Applied Analysis, vol. 2014, Article ID 969265, 5 pages, 2014.

[6] K. I. Beidar and M. A. Chebotar, "On Lie derivations of Lie ideals of prime algebras," Israel Journal of Mathematics, vol. 123, pp. 131-148, 2001. 
[7] W.-S. Cheung, "Lie derivations of triangular algebras," Linear and Multilinear Algebra, vol. 51, no. 3, pp. 299-310, 2003.

[8] F.-Y. Lu, "Lie triple derivations on nest algebras," Mathematische Nachrichten, vol. 280, no. 8, pp. 882-887, 2007.

[9] E. C. Posner, "Derivations in prime rings," Proceedings of the American Mathematical Society, vol. 8, pp. 1093-1100, 1957.

[10] X.-F. Qi and J.-C. Hou, "Characterization of Lie derivations on von Neumann algebras," Linear Algebra and Its Applications, vol. 438, no. 1, pp. 533-548, 2013.

[11] M. N. Daif, "When is a multiplicative derivation additive?" International Journal of Mathematics and Mathematical Sciences, vol. 14, no. 3, pp. 615-618, 1991.

[12] W. Yu and J.-H. Zhang, "Nonlinear Lie derivations of triangular algebras," Linear Algebra and Its Applications, vol. 432, no. 11, pp. 2953-2960, 2010.

[13] P.-S. Ji, R.-R. Liu, and Y.-Z. Zhao, "Nonlinear Lie triple derivations of triangular algebras," Linear and Multilinear Algebra, vol. 60, no. 10, pp. 1155-1164, 2012.

[14] C.-J. Li and X.-C. Fang, "Lie triple and Jordan derivable mappings on nest algebras," Linear and Multilinear Algebra, vol. 61, no. 5, pp. 653-666, 2013.

[15] R. V. Kadison and J. R. Ringrose, Fundamentals of the Theory of Operator Algebras, Vol. I, Academic Press, New York, NY, USA, 1983.

[16] C. R. Miers, "Lie homomorphisms of operator algebras," Pacific Journal of Mathematics, vol. 38, pp. 717-735, 1971.

[17] M. Brešar and C. R. Miers, "Commutativity preserving mappings of von Neumann algebras," Canadian Journal of Mathematics, vol. 45, no. 4, pp. 695-708, 1993.

[18] D. C. Kleinecke, “On operator commutators," Proceedings of the American Mathematical Society, vol. 8, pp. 535-536, 1957.

[19] Z.-F. Bai and S.-P. Du, "Strong commutativity preserving maps on rings," Rocky Mountain Journal of Mathematics, in press. 


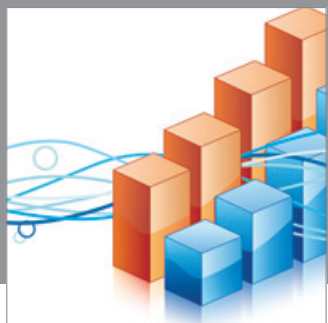

Advances in

Operations Research

mansans

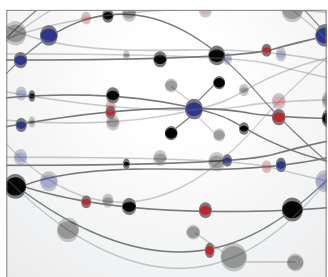

The Scientific World Journal
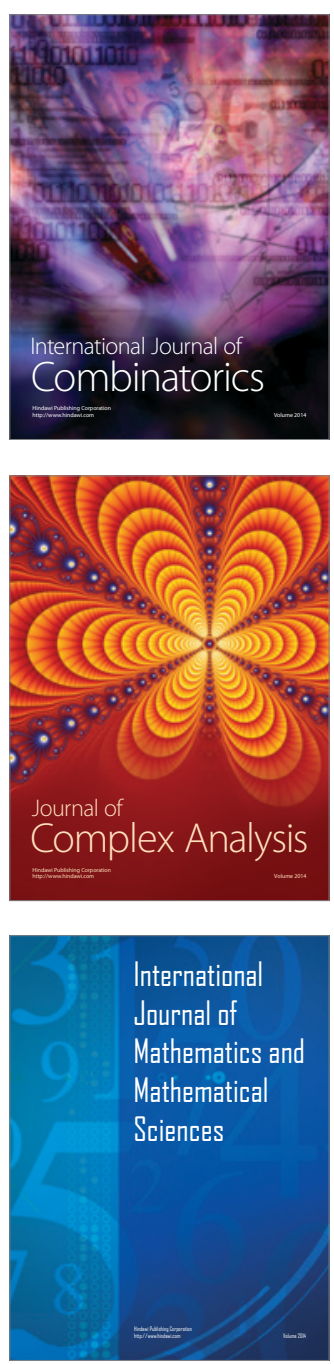
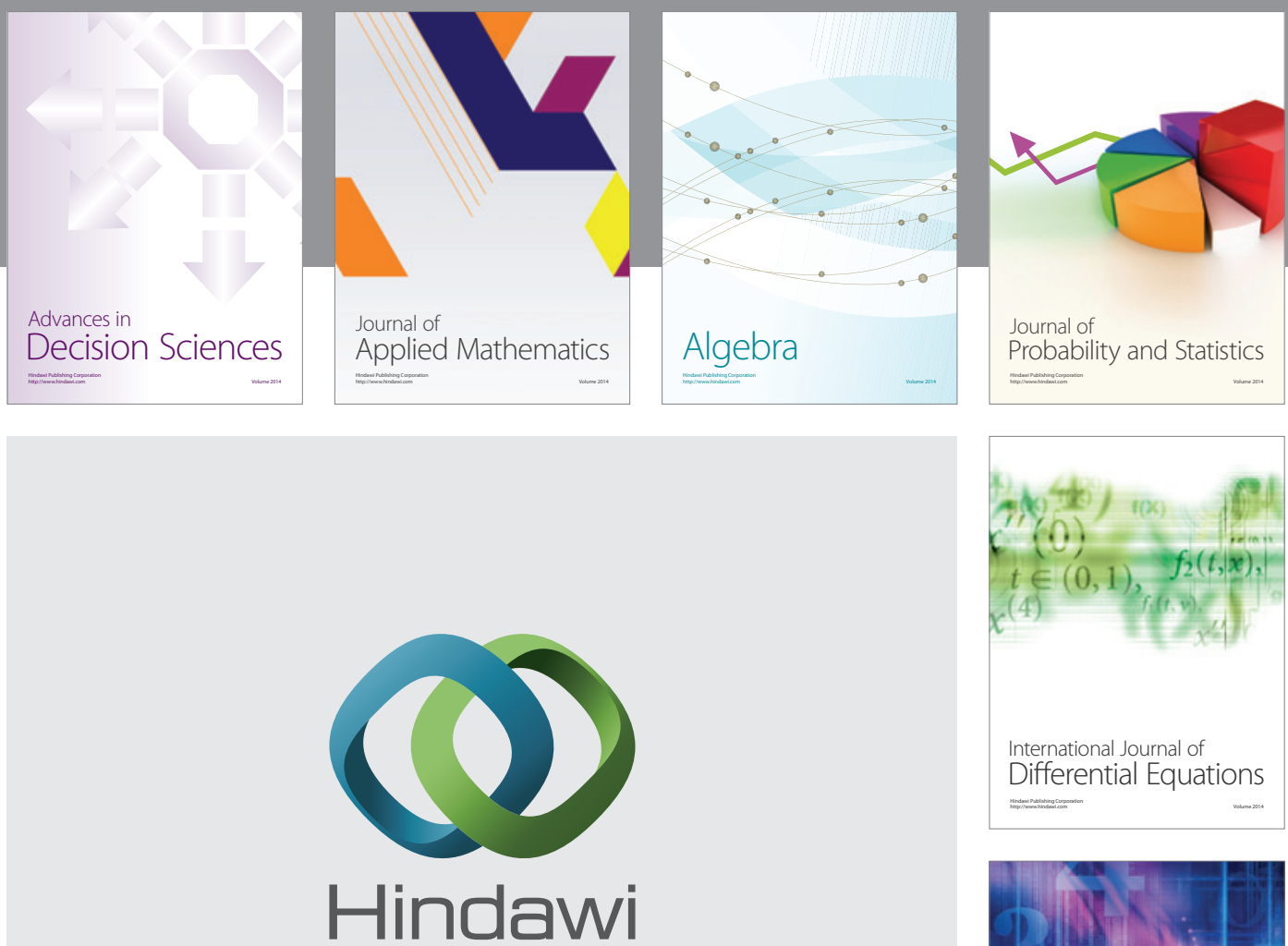

Submit your manuscripts at http://www.hindawi.com
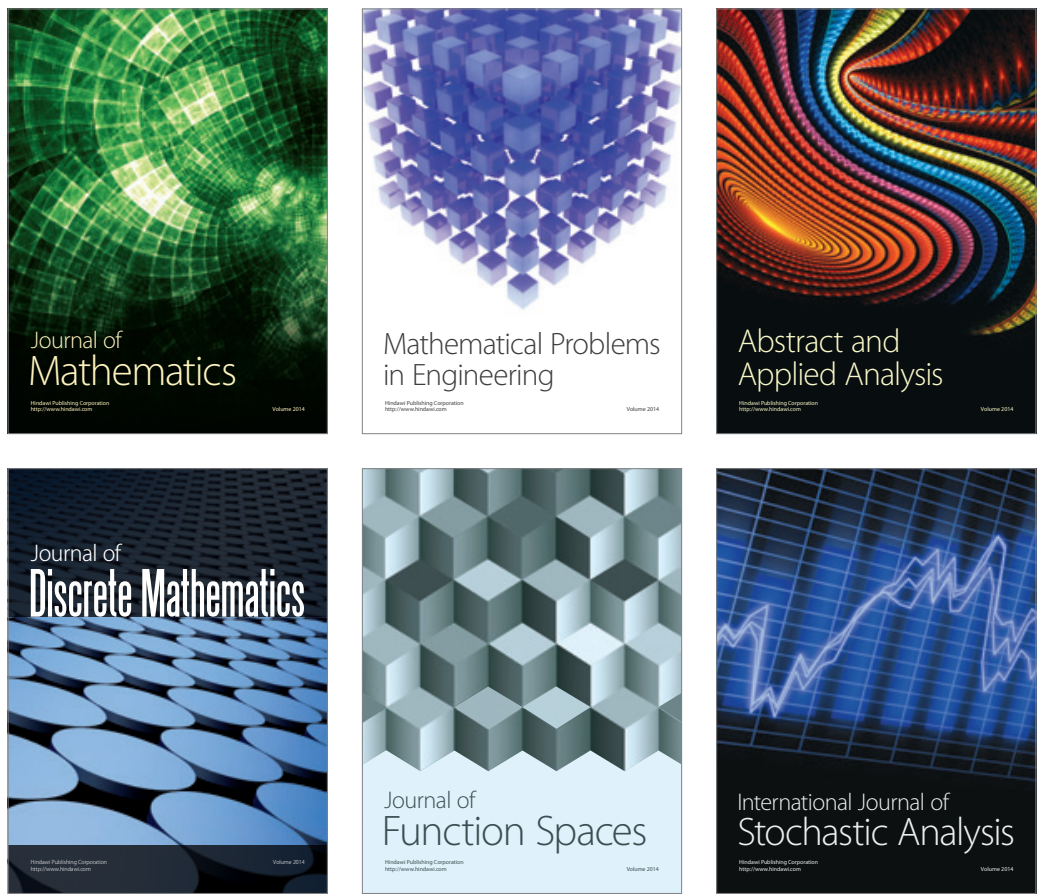

Journal of

Function Spaces

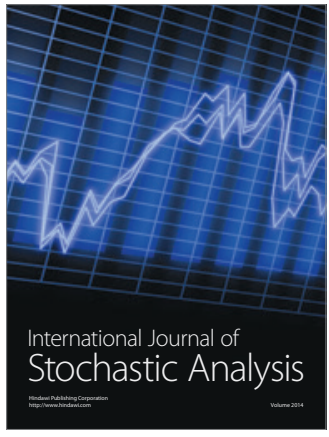

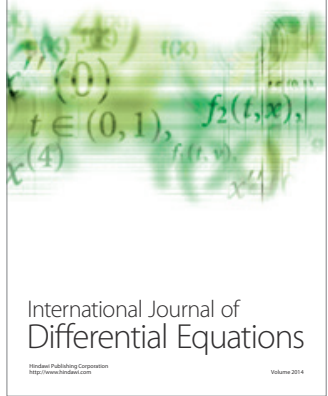
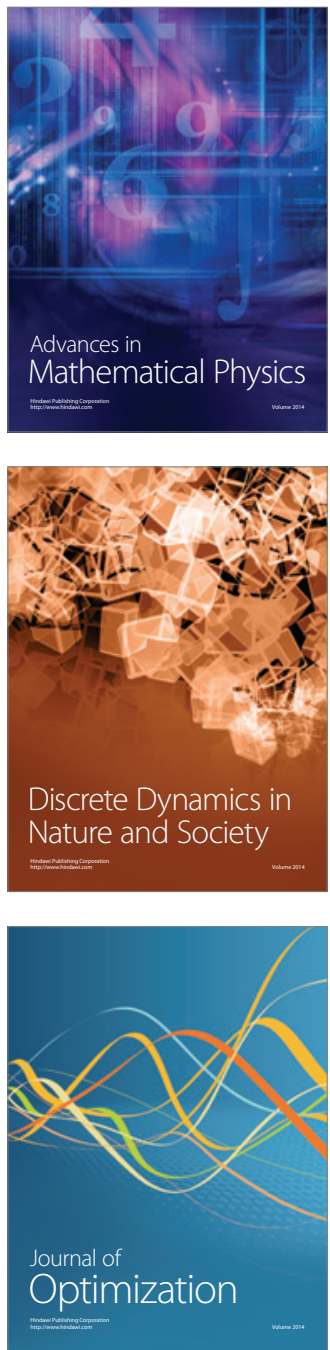